\title{
Die Weiterbildungsbeteiligung von Geringqualifizierten im internationalen Vergleich - Eine Mehrebenenanalyse
}

\author{
Andreas Martin • Ina E. Rüber
}

Eingegangen: 2. Mai 2016 / Angenommen: 28. Juni 2016 / Online publiziert: 27. Juli 2016

(C) Der/die Autor(en) 2016. Dieser Artikel ist eine Open-Access-Publikation.

Zusammenfassung Weiterbildung steht zunehmend im Fokus bildungspolitischer Steuerungsansprüche auf nationaler sowie supra- und internationaler Ebene. International-vergleichende Large-Scale-Assessments wie PIAAC haben die öffentliche Aufmerksamkeit für die Erwachsenen- und Weiterbildung weiter gesteigert und dabei auch die Geringqualifizierten fokussiert. Der Beitrag analysiert anhand von Mehrebenenmodellen die Relevanz nationaler Kontexte für die Weiterbildungsbeteiligung insgesamt und von Geringqualifizierten insbesondere. Unter Benutzung der Daten des European Labour Force Survey aus dem Jahr 2013 wird gezeigt, dass die Höhe nationaler Bildungsausgaben einen positiven Einfluss auf die individuelle Teilnahme an Weiterbildung hat und die Benachteiligung Geringqualifizierter bei höheren Bildungsausgaben geringer ist.

Schlüsselwörter Weiterbildung · Erwachsenenbildung · Internationaler Vergleich · Steuerung · Grundbildung

\section{The participation in further education of low educated in international comparison - a multi-level analysis}

\footnotetext{
Abstract Adult education increasingly occupies the center of education policy and hence the corresponding governance aspirations on the national, supra- and international level. Large-scale-assessment studies, like PIAAC, further raised public attention for adult education with a focus on the low educated. This study analyzes

A. Martin $(\bowtie) \cdot$ I. E. Rüber

Deutsches Institut für Erwachsenenbildung - Leibniz-Zentrum für Lebenslanges Lernen e. V., Bonn, Deutschland

E-Mail: Martin@die-bonn.de

I. E. Rüber

E-Mail: Rueber@die-bonn.de
} 
overall and spatial participation in adult education in the national context using multi-level modeling. Using data from the European Labour Force Survey 2013 it shows that (1) the amount of national educational expenditure is positively correlated with participation in adult education, (2) the disadvantage of low qualified decreases with increasing educational expenditure.

Keywords Adult education - Further education - Continuous education · International comparison · Basic education · Governance

\section{Einleitung}

Weiterbildung steht zunehmend im Fokus bildungspolitischer Steuerungsansprüche von Akteuren auf nationaler sowie supra- und internationaler Ebene. Die vielfältigen Gründe für dieses Interesse lassen sich auf zwei zentrale Punkte zurückführen. Zum einen sind Bildung und Lebenslanges Lernen immer wichtiger werdende Produktions- und Standortfaktoren im internationalen Wettbewerb (vgl. Kulke 2013; Peters et al. 2014), zum anderen wird Bildung - vor allem über die Arbeitsmärkte vermittelt - immer mehr zum Schlüssel und zur Legitimation der Verteilung von Lebenschancen und gesellschaftlicher Teilhabe (vgl. Solga 2005). Im Zentrum politischer Interessen stehen dementsprechend Aspekte der Effektivität und der Effizienz des Bildungssystems, z. B. im Blick auf vermittelte Kompetenzen und Abschlüsse einerseits und gleiche Zugangschancen für alle Bevölkerungsgruppen andererseits.

Die nationalen Zielgrößen bildungspolitscher Interventionen in der Weiterbildung richten sich zunehmend nach internationalen Benchmarks, die besonders von der OECD (z. B. PIAAC, vgl. Rammstedt 2013) und von der Europäischen Union (EU) definiert werden. So strebt die EU für 28 europäische Staaten bis 2020 eine durchschnittliche Weiterbildungsbeteiligungsquote der Bevölkerung zwischen 25 und 65 Jahren von $15 \%$ an. Datengrundlage ist dabei der European Labour Force Survey (EU-LFS) (vgl. EU 2011). Besonderes Augenmerk legt die EU dabei auf die Partizipation benachteiligter Gruppen wie Geringqualifizierte, Ältere und Migranten (vgl. ebd.). Auch auf nationaler Ebene werden konkrete Zielgrößen formuliert (vgl. BMBF 2008; Bilger und Kuper 2013).

Die Umsetzung der bildungspolitischen Zielsetzungen stellt die Akteure vor erhebliche Herausforderungen. Besonders der Bereich der Weiterbildung ist durch ein hohes Maß an Heterogenität auf allen Handlungsebenen geprägt und entzieht sich weitgehend direkten Eingriffen (bildungs-)politischer Akteure. Es setzt sich darum in zunehmendem Maße die Einsicht durch, dass die Wirkungen organisierter Weiterbildung das Ergebnis von Angeboten und deren Nutzung ist, die sich aus der Ko-Konstruktion vielfältiger Akteure auf unterschiedlichen Handlungsebenen ergeben (vgl. Schrader 2008). Im Zuge des neuen, an Dezentralisierung und (Teil-)Autonomie orientierten Steuerungsparadigmas in der (Weiter-)Bildung, richtet sich die Aufmerksamkeit dabei zunehmend auf Konzepte, die bildungsrelevante Akteurskonstellationen in räumlichen Aggregaten zusammenfassen und diese ,vor Ort" koordinieren. Im Zentrum dieser Bemühungen stehen vor allem kleinräumige regionale und kommunale Bezugsrahmen. Der Einfluss dieser räumlichen Kontexte 
und Regulative auf das Weiterbildungsverhalten der Bevölkerung und spezifischer, benachteiligter Bevölkerungsgruppen sowie die sich daraus ergebenen Steuerungspotenziale, haben sich bisher jedoch als überschaubar erwiesen (vgl. Martin und Schömann 2015; Martin et al. 2016). Die Ergebnisse legen die Vermutung nahe, dass sich die entscheidenden wirtschaftlichen, kulturellen und sozialen Kontexte und die bildungspolitischen Regulative des Weiterbildungsverhaltens weniger entlang regionaler oder kommunaler Räume differenzieren, sondern vor allem auf der Ebene von Nationen variieren. Dies ist jedoch nur im internationalen Vergleich messbar.

Folglich stellt sich die Frage, welchen Einfluss nationale Kontexte und Regulative auf die Weiterbildungsbeteiligung tatsächlich haben und inwiefern es auf dieser Handlungsebene gelingt, die Partizipation von Bevölkerungsgruppen, deren Beteiligungschancen durch typische Barrieren eingeschränkt sind, zu erhöhen und so soziale Selektivität im Bildungsverhalten abzubauen. Wir untersuchen diese Fragestellung anhand der Daten des EU-LFS 2013 für den europäischen Raum.

\section{Partizipationsstrukturen der Weiterbildung - individuelle und kontextuelle Prädiktoren}

\subsection{Individuelle Merkmale und Barrieren}

Die Partizipation an Weiterbildung hängt in hohem Maße von Merkmalen der individuellen Lebenslage und des unmittelbaren Kontextes - wie die Familie oder der Betrieb - ab. Auf Basis des Adult Education Survey (AES) haben sich auf individueller Ebene vor allem sozioökonomische (Schulbildung, berufliche Ausbildung) und demografische Merkmale (Alter, Geschlecht, Migrationshintergrund) als stark prädiktiv erwiesen (vgl. Kuper et al. 2013). Die Analyse des Mikrozensus in Deutschland zeigt, dass darüber hinaus der Familienstand und die Zahl der Kinder (besonders bei Alleinerziehenden) relevant sind. Auch die Branche und das verfügbare Haushaltseinkommen wirken sich auf die Weiterbildungsbeteiligung aus (vgl. Martin et al. 2015). Weitere wichtige Einflussfaktoren ergeben sich aus der Betriebszugehörigkeit und den Merkmalen dieses unmittelbaren Kontextes (vgl. Schiener et al. 2013). Alter, Geschlecht, Bildungsstand und Arbeitsmarktstatus behalten auch über die nationalen Grenzen hinaus Relevanz, wie die international vergleichende Forschung bestätigt (vgl. Wolbers 2005; Dämmrich et al. 2014).

Mit den individuellen Merkmalen verbinden sich in einigen Fällen auch typische strukturelle, situationelle und dispositionale Barrieren der Weiterbildungsbeteiligung (vgl. Cross 1981). Besonders häufig werden diese in Bezug auf Geringqualifizierte diskutiert. Hier beeinträchtigen vor allem dispositionale, aber auch strukturelle Barrieren die Weiterbildungsbeteiligung. Geringer Status und unsichere Lebensbedingungen führen häufiger zu einer kurzfristigen, nicht an beruflicher Entwicklung ausgerichteten Lebensplanung. Damit entfallen wichtige Anreize für die Weiterbildungsbeteiligung (vgl. Reutter 2011). Dies gilt auch für die meist einfachen Tätigkeiten ohne Anregungspotenzial (vgl. Siebert 2006). Mit niedrigen Bildungsabschlüssen verbinden sich zudem in der Regel auch niedrige Kompetenzniveaus. Bei höheren Bildungsanforderungen führt dies häufiger zu Erfahrungen der Überforderung (vgl. 
ebd.). Strukturelle Barrieren ergeben sich für Geringqualifizierte besonders im Bereich der betrieblichen Weiterbildung (vgl. Ericson 2005).

Auch wir fokussieren diese benachteiligte Gruppe und rekurrieren dabei besonders auf die dispositionalen Barrieren. Wir gehen zunächst davon aus, dass geringe Qualifikationen auch im internationalen Vergleich zu einer geringeren Weiterbildungsbeteiligung führen und formulieren die anhand anderer Datengrundlagen bereits bestätigte Hypothese:

H1 Die Teilnahmewahrscheinlichkeit an Weiterbildung ist bei Personen mit niedrigem Bildungsstand geringer als die Teilnahmewahrscheinlichkeit von Personen mit höherem Bildungsstand.

\subsection{Länderspezifische Merkmale}

Bedingt durch die eingeschränkte Verfügbarkeit entsprechender Daten erweisen sich im Gegensatz zu den Einflussfaktoren auf der individuellen Ebene Untersuchungen zum Einfluss von Merkmalen höherer räumlich zusammengefasster Aggregate als schwierig. Zum Einfluss nationaler Kontexte und Regulative wurde eine Reihe theoretischer Annahmen formuliert und anhand internationaler Datensätze untersucht. Dabei wird deutlich, dass sich - anders als auf kommunaler und regionaler Ebene - mit den Ländergrenzen Systeme unterscheiden lassen, in denen sich spezifische Konstellationen des Zusammenwirkens wirtschaftlicher, politischer, sozialer und kultureller Prozesse etabliert haben. In Bezug auf das Weiterbildungsverhalten dominieren zwei theoretische Ansätze: die Wohlfahrtsstaatendifferenzierung nach Esping-Andersen (1990) und die Variationen des Kapitalismus nach Hall und Soskice (2001).

Ausgangspunkt der Überlegungen zur Wohlfahrtsstaatendifferenzierung ist die Annahme, dass die durch den Status eines Bürgers bezeichnete Gleichheit sozialer Rechte mit dem Prinzip der marktvermittelten Klassenlagen und der sich daraus ergebenen faktischen Ungleichheit kollidiert (vgl. Esping-Andersen 1990, S. 36). Esping-Andersen (1990) entwickelt drei idealtypische Wohlfahrtsstaatsregime (liberal, korporatistisch und sozialdemokratisch), unterschieden durch den Grad der De-Kommodifizierung und der Bedeutung von Markt, Staat und Familie. In liberalen Wohlfahrtsregimen (US, CA, AU) wird die Marktabhängigkeit der Individuen kaum durch De-Kommodifizierung von Leistungen eingeschränkt. Die hier dominierende Form bedarfsorientierter Unterstützung stellt zwar ein soziales Netz dar, ist jedoch aufgrund des geringen Leistungsumfanges und der damit einhergehenden Stigmatisierung wenig geeignet, die Abhängigkeit der Individuen vom Markt aufzuheben. Ungleichheit nimmt in diesem Regimetyp tendenziell zu. Auf den Bereich der Weiterbildung übertragen bedeutet dies, dass Weiterbildungsaktivitäten über den Markt organisiert und privat finanziert werden. Öffentliche Finanzierung kommt nur in nachgewiesenen Bedürftigkeitsfällen infrage und bleibt die Ausnahme (vgl. Kaufmann et al. 2014).

Korporatistische Wohlfahrtsstaatsregime (DE, FR, IT, AT) orientieren sich stark am Subsidiaritätsprinzip und verknüpfen Ansprüche auf Sozialleistungen mit vorher erbrachten Beiträgen. Eine wichtige Rolle spielen dabei wert- und interessengelei- 
tete Gemeinschaften wie Innungen, Berufsverbände, Kammern und Kirchen. Dieses Wohlfahrtsregime unterstützt die Reproduktion sozialer Ungleichheit. Weiterbildung steht für einzelne Statusgruppen in unterschiedlichem Maße zur Verfügung und wird nur in Form von Zuschüssen durch den Staat unterstützt (vgl. ebd.).

In sozialdemokratischen Wohlfahrtsstaatsregimen (NO, SE) werden aus einem universalistischen Prinzip heraus gleiche soziale Rechte für alle Bürger definiert und in Form weitgehend vorrausetzungsloser Ansprüche auf Sozialleistungen umgesetzt. Das hohe Maß an De-Kommodifizierung führt zu einer Verringerung sozialer Ungleichheit. Weiterbildung wird auf hohem Niveau durch den Staat finanziert und steht allen Bürgern weitgehend voraussetzungslos zur Verfügung (vgl. ebd.).

Versuche, eine größere Anzahl von Ländern den einzelnen Wohlfahrtsregimen zuzuordnen, verdeutlichen zumeist die Differenz zwischen Idealtypus und empirischer Wirklichkeit. Esping-Andersen (vgl. 1999) selbst revidiert die ursprüngliche Klassifizierung und bezeichnet Länder wie die Schweiz oder Belgien als hybride Typen. Staaten des ehemaligen Ostblocks und Länder, deren De-Kommodifizierungsmechanismen nicht durch die beschriebenen Formen sozialen Ausgleichs, sondern über Arbeitsmarktmaßnahmen funktionieren, werden als eigener Typus vorgeschlagen (vgl. Castles und Mitchell 1993; Castles und Obinger 2008). Die Zuordnung der Länder zu den Kategorien ist in der Literatur bis heute nicht kohärent (vgl. Ebbinghaus 2012). Dennoch werden modifizierte Klassifikationen der Wohlfahrtsstaatregime vielfach als analytische Grundlage des Einflusses nationaler Merkmale auf das Weiterbildungsverhalten genutzt (vgl. Boeren et al. 2012; Roosmaa und Saar 2012; Markowitsch et al. 2013; Boeren und Holford 2016).

Der zweite theoretische Ansatz der Variationen des Kapitalismus (vgl. Hall und Soskice 2001) folgt der Grundannahme, dass Firmen die zentralen Akteure in einer Ökonomie sind und Koordinationsleistungen in fünf Bereichen erbringen: (1) die Verhandlungen über Gehälter, Einkommen und Arbeitsbedingungen in Koordination mit Gewerkschaften und anderen Formen organisierter Interessenvertretungen, (2) die Aufrechterhaltung und Steigerung von Kompetenzen der Arbeitskräfte in Absprache mit den Arbeitnehmern, (3) der Umgang mit Investoren und deren Renditeerwartungen, (4) die Beziehungen zu Zulieferfirmen, Großkunden und PartnerFirmen und schließlich (5) der Ausgleich der Interessen der Belegschaft und der Firma.

Aus der für eine Gesellschaftsformation typischen Art und Weise, wie Firmen diesen fünf Koordinationsanforderungen gerecht werden, leiten sich zwei Typen des Kapitalismus ab, die als liberale und koordinierte Marktökonomien bezeichnet werden. Sie bilden Pole einer stetigen Dimension von mehr oder weniger marktgesteuerten Koordinationsformen. In liberalen Marktökonomien orientieren sich die Firmen bei ihren Koordinationsleistungen am Markt und nutzen interne Hierarchien. Im Zentrum stehen dabei rationale Entscheidungsfindungen in Abwägung von Angebot und Nachfrage. Der politische Überbau dieser Gesellschaftsformationen korrespondiert mit der marktförmigen Ausrichtung betrieblicher Koordinationsformen und zeichnet sich vor allem durch ein hohes Maß an Deregulierung aus. Die Marktorientierung impliziert in Bezug auf die Weiterbildung vor allem aufseiten der Arbeitnehmer ein Interesse an allgemein verwertbarer, betriebsunspezifischer Bildung (vgl. Kaufmann et al. 2014). In koordinierten Marktwirtschaften werden 
die Koordinationsleistungen hingegen weniger anhand des Marktes, sondern vor allem in Form strategischer Partnerschaften und Netzwerke organisiert. Dies ermöglicht es den Firmen, technologische Fähigkeiten auszubauen, das eigene Profil zu schärfen und sich zu spezialisieren. Weiterbildung ist hier in höherem Maße an betriebs- und berufsspezifischen Qualifikationen orientiert (vgl. ebd.). Auch diese Systematik wurde in einer Vielzahl empirischer Untersuchungen zum Einfluss nationaler Kontexte auf das Weiterbildungsverhalten angewandt. So untersuchen Roosmaa und Saar (2012) anhand einer Kombination der dominierenden Koordinationsform und der Ausprägung des Wohlfahrtsstaates den Einfluss makrostruktureller Merkmale auf die Bedeutung von Angebot und Nachfrage nach Qualifikationen auf dem Arbeitsmarkt für die Weiterbildungsbeteiligung. Sie zeigen, dass besonders in marktkoordinierten Gesellschaften die Nachfrageseite in hohem Maße Einfluss auf die Weiterbildungsbeteiligung von Geringqualifizierten und auf die Selektivität des Weiterbildungssystems nimmt. Auch in anderen Untersuchungen werden Variationen des Kapitalismus zur theoretischen Operationalisierung makrostruktureller Merkmale herangezogen (vgl. Dieckhoff et al. 2007; Roosmaa und Saar 2012; Desjardins und Rubenson 2013).

Die Literatur zeigt zudem, dass nationale Kontexte Einfluss auf das Weiterbildungsverhalten haben. Dies wird an einer Reihe von Makro-Merkmalen wie bspw. der Gewerkschaftsdichte, der Ausgaben für Forschung und Entwicklung, den Sozialausgaben und Ausgaben für Bildung deutlich (vgl. Bassanini et al. 2005; Dämmrich et al. 2014). Wie groß jedoch der Einfluss der nationalen Ebene auf das Weiterbildungsverhalten insgesamt ist, wissen wir bisher nicht. Wir gehen davon aus, dass der Varianzanteil der nationalen Ebene an der Weiterbildungsbeteiligung deutlich höher ist als dies etwa für regionale und kommunale Raumabgrenzungen beobachtet werden konnte (ca. 4 \%, vgl. Martin und Schömann 2015).

\subsection{Interaktionen zwischen individuellen und länderspezifischen Merkmalen}

Der Einfluss länderspezifischer Kontexte und Regulative auf die Weiterbildungsbeteiligung ist ohne eine konkrete Annahme, wie diese strukturellen Bedingungen auf das individuelle Handeln einwirken, nicht denkbar. Dazu scheint es zunächst notwendig, die individuelle und die nationalstaatliche Handlungsebene sowie die auf diesen Ebenen relevanten Akteure in das System der Weiterbildung einzuordnen. Dazu bietet sich das Mehrebenensystem der Weiterbildung nach Schrader (vgl. 2008, 2011) an. In diesem Mehrebenenmodell lassen sich die Ebenen der supranationalen Bildungspolitik, der nationalen Bildungspolitik, der institutionellen Umwelt von Organisationen, der Organisationen selbst und schließlich die Handlungsebene der Lehr-Lernsituationen unterscheiden. Zentral für die Frage nach bildungspolitischen Einflussmöglichkeiten sind in diesem Konzept vor allem drei Punkte (vgl. Martin et al. 2016):

Zum einen steht die Nutzung von Angeboten im Mittelpunkt der Ebene der LehrLernprozesse und damit im Zentrum des Weiterbildungssystems. Alle Aktivitäten von Akteuren auf anderen Handlungsebenen sind letztlich auf das Zustandekommen und die wirksame Gestaltung von Lernaktivitäten gerichtet. Da die Teilnahme die Bedingung der Möglichkeit für jeden Lehr-Lernprozess und dessen wirksame 
Gestaltung ist, kann der (Nicht-)Teilnehmende als ein zentraler Akteur des Weiterbildungssystems aufgefasst werden. Eine zweite wichtige Annahme des Modells bezieht sich auf die wechselseitige Beeinflussung von Akteuren auf unterschiedlichen Handlungsebenen durch die Veränderung des jeweiligen Handlungskontextes. Dies legt im Falle der nationalen Ebene und der Handlungsebene des Lehr-Lernprozesses nahe, dass nicht nur die Akteure der nationalen Ebene (Staat, Länder, Regionen, Kommunen) das Handeln von Akteuren der Handlungsebene der LehrLernsituationen beeinflussen, sondern dass auch die Akteure dieser untersten Handlungsebene Einfluss auf die Bildungspolitik der nationalen Ebene nehmen können. Schließlich geht das Modell davon aus, dass eine wechselseitige Beeinflussung der Handlungen nur dann möglich ist, wenn die Eingriffe in den Handlungskontext von Akteuren durch diese re-kontextualisiert, also zum Bestandteil der jeweiligen Handlungsorientierung gemacht werden.

Das Modell lässt jedoch offen, wie genau diese wechselseitige Beeinflussung von Kontexten durch Akteure der nationalen Ebene und der Ebene der Lehr-Lernprozesse sattfinden kann und wie eine Re-kontextualisierung aussieht.

Rubenson und Desjardins (2009) bieten ein theoretisches Konzept, das BoundedAgency-Modell, welches an diesem Punkt anschlussfähig ist. Ausgangspunkt der Überlegungen ist die Frage, wie sich strukturelle Merkmale, welche sich sowohl auf unterschiedliche Wohlfahrtsregime (vgl. ebd.) als auch auf Variationen des Kapitalismus (vgl. Desjardins und Rubenson 2013) zurückführen lassen, auf das individuelle Handeln auswirken und wie das Handeln wiederum die strukturellen Merkmale beeinflusst. In Anlehnung an den Neostrukturalismus Bourdieus (1990) beantworten die Autoren die Fragestellung anhand der Wechselwirkung von dispositionalen und strukturellen Barrieren der Weiterbildungsbeteiligung. Die zentrale Modifikation der Bounded-Agency-Theorie gegenüber den Konzepten der Wohlfahrtsregime und den Variationen des Kapitalismus besteht darin, dass diese Gesellschaftsformationen praxistheoretisch interpretiert werden. Wohlfahrtsregime sind danach nicht nur Ausdruck struktureller Eigenarten einer Gesellschaft, sondern vermittelnde Praxis zwischen Habitus und sozialem Feld. Die in einer wohlfahrtsstaatlichen Praxis typischen Strukturen korrespondieren mit entsprechenden Dispositionen (Habitus) und reproduzieren sich im Vollzug des Handelns wechselseitig. Die Autoren gehen dabei davon aus, dass Strukturen sowohl Werte als auch das Bewusstsein des Individuums für Möglichkeiten bedingen. Bounded-Agency ist die dispositional bedingte und strukturell eingeschränkte individuelle Handlung. Strukturelle Barrieren ergeben sich aus den realisierten, für bestimmte Gesellschaftsformationen typischen Einschränkungen von Handlungsoptionen. Dispositionale Barrieren hingegen ergeben sich aus den inkorporierten und erlebten Einschränkungen von Handlungsmöglichkeiten.

Anhand der Annahmen der Bounded-Agency-Theorie ergeben sich Implikationen zu den Steuerungspotenzialen bildungspolitischer Akteure auf der Ebene der Nationalstaaten:

Public policy as represented by different types of welfare state regimes can directly affect the contextual (structural) conditions that individuals face (...) but through this it can also indirectly affect individuals' subjective rationality and 
view (disposition) of their opportunity structure. Only through this structureagency interaction does policy affect the take-up of learning (vgl. Rubenson und Desjardins 2009, S. 196).

Das Bounded-Agency Modell geht davon aus, dass Barrieren der Weiterbildungsbeteiligung Handlungspraxen darstellen, in welchen handlungseinschränkende Strukturen und Dispositionen durch Rückkopplungsprozesse ein Gleichgewicht ansteuern und schließlich korrespondieren. Darin liegt auch das entscheidende Steuerungspotenzial nationaler Bildungspolitik. Unterschiedlich ausgeprägte strukturelle Barrieren in verschiedenen Ländern gehen demnach auch mit unterschiedlich ausgeprägten Dispositionen einher. Dies sollte im Weiterbildungsverhalten der jeweiligen Wohnbevölkerung sichtbar werden. Ein wichtiger Einflussfaktor für den allgemeinen Umfang von Gelegenheitsstrukturen in einem Land und die Weiterbildungsbeteiligung sind die öffentlichen Ausgaben für Bildung (vgl. Groenez et al. 2007). Diese Kennzahl nutzen wir in Form von öffentlichen Bildungsausgaben pro Einwohner, welche zum einen die zugeschriebene Relevanz von Bildung abbildet (vgl. Dämmrich et al. 2014, S. 38) und zum anderen die beste Annäherung an den tatsächlichen Gelegenheitsumfang im Erfahrungsbereich des Individuums darstellt. Somit sollte diese Kennzahl mittelfristig mit einem entsprechenden Verhalten korrespondieren:

H2 Je höher die öffentlichen Bildungsausgaben innerhalb eines Landes, desto größer ist die Wahrscheinlichkeit der Weiterbildungsbeteiligung der in diesem Land lebenden Bevölkerung.

Wie Hypothese 1 ist auch Hypothese 2 eine Voraussetzung für die nachfolgende Fragestellung.

Die Wechselwirkung von strukturellen und dispositionalen Barrieren sollte besonders deutlich am Einfluss von Gelegenheitsstrukturen auf das Weiterbildungsverhalten von Personengruppen sichtbar werden, deren Weiterbildungsbeteiligung vor allem durch dispositionale Barrieren eingeschränkt ist (hier: Geringqualifizierte). Öffentliche Bildungsausgaben sollten auch die Weiterbildungsbeteiligung dieser Personengruppe erhöhen. Legt man dabei - wie es Rubenson und Desjardins (2013) tun - die Logik der Wohlfahrtsstaatsregime zugrunde, wonach de-kommodifizierende Aktivitäten des Staates der Kumulation von Ungleichheiten in marktvermittelten Zusammenhängen entgegenwirken, sollte zudem mit höheren öffentlichen Bildungsausgaben eine Abnahme der Ungleichverteilung von Bildungschancen einhergehen. Entsprechend gehen wir zunächst davon aus, dass sich die Effektstärke von niedriger Bildung zwischen den Ländern unterscheidet. Weiter nehmen wir an, dass hohe Bildungsausgaben strukturelle und dispositionale Barrieren minimieren und die Ungleichheit von Beteiligungschancen reduzieren:

H3 Je höher die öffentlichen Bildungsausgaben in einem Land, desto geringer ist der negative Einfluss von niedriger Bildung auf die Weiterbildungsbeteiligung. 


\subsection{Daten und Methoden}

Die empirische Prüfung der theoretischen Überlegungen erfordert eine Datengrundlage, in der individuelle Merkmale erfasst und in nationale Kontexte genestet sind. Diesen Ansprüchen wird der seit 1983 erhobene European Labor Force Survey (EU-LFS) gerecht. Mit Schwerpunkt auf die Messung von Arbeitsmarktkennzahlen werden im EU-LFS vierteljährlich Daten zu zentralen sozioökonomischen und demografischen Merkmalen der Wohnbevölkerung europäischer Staaten im Alter ab 15 Jahren anhand von Haushaltsbefragungen erfasst. Das gesamte Erhebungsverfahren ist stark standardisiert, um die Vergleichbarkeit der Daten über Ländergrenzen hinweg sicherzustellen. Der anhand des EU-LFS erzeugte Indikator zum Lebenslangen Lernen wird im Rahmen der Lissabon-Strategie von der EU als Benchmark genutzt (vgl. Behringer und Schönfeld 2014). Dennoch gibt es auch hier Einschränkungen. Zum einen werden im EU-LFS wie auch im Mikrozensus Proxy-Interviews genutzt, zum anderen ist die Operationalisierung der Weiterbildungsbeteiligung in den Ländern nicht immer identisch (vgl. ebd.). Für unsere Analysezwecke nutzen wir das Jahres-Datenfile 2013, welches den Durchschnitt der vierteljährig erhobenen Daten aus 2013 enthält. Mit 4.645.916 Fällen in 31 europäischen Ländern steht damit die derzeit breiteste Datengrundlage für eine international vergleichende Untersuchung des Weiterbildungsverhaltens zur Verfügung. Die für dieses räumliche Aggregationsniveau relativ hohe Zahl an Clustern (Ländern) stellt eine hinreichende Grundlage für Mehrebenenanalysen dar (vgl. Langer 2009). Vor allem aufgrund dieses Vorteils ziehen wir hier den LFS anderen Statistiken wie etwa dem AES vor.

Grundgesamtheit unserer Untersuchung ist die Wohnbevölkerung im Alter zwischen 22 und 67 Jahren. Die ungewöhnliche Altersbegrenzung ist auf die in Intervallen von fünf Jahren zusammengefassten Angaben im Scientific Use File zurückzuführen. Wir schließen jüngere Befragte aus den Analysen aus, da uns die Weiterbildungsbeteiligung Erwachsener, die eine Erstausbildung absolviert haben, interessiert. Eine aktuelle Teilnahme an anderen, formalen Bildungsgängen ist jedoch möglich. Abzüglich der Fälle mit fehlenden Angaben beläuft sich die Fallzahl damit auf 2.550.763 in 30 Ländern. Griechenland wurde aus der Untersuchung ausgeschlossen, da keine Informationen zu den dortigen Bildungsausgaben zur Verfügung stehen.

Tab. 1 beinhaltet die Deskription unserer Stichprobe im Blick auf die für unsere Modelle relevanten Variablen. Bei der abhängigen Variable handelt es sich um die Auskunft zur Beteiligung im Bereich der non-formalen Bildung in den letzten vier Wochen: „Did you attend any courses, seminars, conferences or received private lessons or instructions outside the regular education system (...) within the last 4 weeks" (vgl. Eurostat 2015, S. 28).

Die zentrale unabhängige Variable auf der Mikroebene ist der Bildungsstand. Dieser orientiert sich an der im LFS bis 2013 verwendeten Klassifikation ISCED 97 zum höchsten bisher erreichten Bildungsabschluss. Geringqualifiziert ist hier, wer keine Berufsausbildung bzw. Abitur (ISCED 3 a und b) oder höhere Abschlüsse aufweist. Die mit geringen Qualifikationen verbundenen dispositionalen Barrieren grenzen wir durch die Kontrolle der Stellung im Beruf, den Jobskills sowie der Branche von arbeitsmarktbezogenen, strukturellen Barrieren ab. 
Tab. 1 Deskriptive Statistik und Kodierung der modellspezifischen Variablen

\begin{tabular}{|c|c|c|c|}
\hline Kategoriale Variablen & $\%$ & SE & $\overline{\operatorname{Min}-M a x}$ \\
\hline Weiterbildung & 7,80 & 0,2682 & $0 / 1$ \\
\hline \multicolumn{4}{|l|}{ Bildung } \\
\hline Niedrig & 43,38 & 0,4956 & $0 / 1$ \\
\hline Mittel & 31,53 & 0,4646 & $0 / 1$ \\
\hline Hoch & 25,09 & 0,4335 & $0 / 1$ \\
\hline Migrant & 10,16 & 0,3021 & $0 / 1$ \\
\hline \multicolumn{4}{|l|}{ Alter } \\
\hline 22 bis 32 & 18,42 & 0,3877 & $0 / 1$ \\
\hline 32 bis 42 & 22,61 & 0,4183 & $0 / 1$ \\
\hline 42 bis 52 & 24,92 & 0,4325 & $0 / 1$ \\
\hline 52 bis 62 & 24,30 & 0,4289 & $0 / 1$ \\
\hline$>62$ & 9,75 & 0,2966 & $0 / 1$ \\
\hline Frau & 51,86 & 0,4997 & $0 / 1$ \\
\hline Ledig/geschieden/verwitwet & 37,66 & 0,4845 & $0 / 1$ \\
\hline Erwerbslos & 6,02 & 0,2378 & $0 / 1$ \\
\hline Selbstständig & 10,10 & 0,3013 & $0 / 1$ \\
\hline Angestellte/r & 53,80 & 0,4986 & $0 / 1$ \\
\hline \multicolumn{4}{|l|}{ Anforderungen im Beruf } \\
\hline Elementar & 5,49 & 0,2278 & $0 / 1$ \\
\hline Durchschnittlich & 32,02 & 0,4666 & $0 / 1$ \\
\hline Hoch & 11,13 & 0,3146 & $0 / 1$ \\
\hline Sehr hoch & 15,16 & 0,3587 & $0 / 1$ \\
\hline \multicolumn{4}{|l|}{ Berufsklassifikation } \\
\hline Land-/Forstwirtschaft, Fischerei \& Bergbau (1) & 4,30 & 0,2028 & $0 / 1$ \\
\hline Verarbeitendes Gewerbe (2) & 10,27 & 0,3036 & $0 / 1$ \\
\hline $\begin{array}{l}\text { Energie- \& Wasserversorgung, Abwasser- \& Abfallentsorgung } \\
\text { (3) }\end{array}$ & 1,05 & 0,1017 & $0 / 1$ \\
\hline Baugewerbe (4) & 4,39 & 0,2048 & $0 / 1$ \\
\hline Handel, Instandhaltung \& Reparatur von KFZ (5) & 8,32 & 0,2762 & $0 / 1$ \\
\hline Gastgewerbe (6) & 2,30 & 0,1500 & $0 / 1$ \\
\hline Verkehr \& Lagerei (7) & 3,35 & 0,1800 & $0 / 1$ \\
\hline Information \& Kommunikation (8) & 1,66 & 0,1277 & $0 / 1$ \\
\hline $\begin{array}{l}\text { Dienstleistungen, Grundstücks-, Wohnungswesen \& öffentl. } \\
\text { Dienst (9) }\end{array}$ & 27,56 & 0,4468 & $0 / 1$ \\
\hline $\begin{array}{l}\text { Private Dienstl., exterritoriale Organisationen \& Körperschaften } \\
\text { (10) }\end{array}$ & 0,71 & 0,0840 & $0 / 1$ \\
\hline Betriebsgröße $>10$ Mitarbeiter & 41,71 & 0,4931 & $0 / 1$ \\
\hline Stetige Variablen & Mittelwert & SE & Min-Max \\
\hline Anzahl Kinder im Haushalt & 0,9150 & 1,9260 & $0-12$ \\
\hline Anzahl Kinder im Alleinerzieher/in-Haushalt & 0,2220 & 1,0199 & $0-12$ \\
\hline Wöchentliche Arbeitszeit & 23,5514 & 19,935 & $0-60$ \\
\hline \multicolumn{4}{|l|}{ Länderebene } \\
\hline Bildungsausgaben & 1.432 .367 & 9.346 .099 & $173-5141,7$ \\
\hline
\end{tabular}

Quelle: EU-LFS 2013; $N=2.550 .763$ Individuen; $N=30$ Länder 
Auf der Makroebene spielen wir der Datengrundlage des LFS die öffentlichen Bildungsausgaben als unabhängige Variable zu. Diese stammen von Eurostat (2016) und beziehen sich auf die Ausgaben des öffentlichen Sektors im Jahr 2012 für Bildung mit Ausnahme der frühkindlichen Bildung. Dies haben wir auf die Ausgaben pro Einwohner umgerechnet. Angaben zu Ausgaben für den Weiterbildungsbereich in diesem Zeitraum für alle untersuchten Länder stehen leider nicht zur Verfügung. Wir wählen die zeitliche Verzögerung von einem Jahr bewusst, da wir davon ausgehen, dass die tatsächliche Wirkung von Bildungsausgaben erst nach einer gewissen Zeit eintritt. Für die analytischen Modelle wurde der Wert zentriert und standardisiert.

Die restlichen in Tab. 1 aufgeführten Variablen sind Kontrollvariablen, die, basierend auf bisheriger Forschung, in starkem Zusammenhang mit der Weiterbildungsbeteiligung stehen.

Zur Überprüfung der Hypothesen wenden wir aufeinander aufbauende Mehrebenenmodelle an. Obwohl die Zahl von 30 Ländern für dieses Vorgehen prinzipiell ausreichend ist, bewegen wir uns dennoch am unteren Rande der notwendigen Voraussetzungen. Die Modellierung wurde deswegen auf der Makroebene (Level 2) auf das Notwendige begrenzt.

Um zu ermitteln, welchen Einfluss die Nationen auf das Weiterbildungsverhalten insgesamt haben, stellen wir zunächst ein einfaches logistisches Random-InterceptModell zur Weiterbildungsbeteiligung über die 30 Länder auf (Tab. 2, Modell 0). Anhand der dort ausgewiesenen Varianzanteile berechnen wir die entsprechende Inter-Class-Correlation (ICC) (vgl. Goldstein et al. 2002). Sie gibt Auskunft über die Ähnlichkeit der Weiterbildungsbeteiligung sozialer Gruppen innerhalb derselben Länder. Um zu prüfen, inwiefern Geringqualifizierte tatsächlich auch im internationalen Vergleich und anhand unserer Datengrundlage geringere Teilnahmewahrscheinlichkeiten aufweisen (H1), nehmen wir die individuelle unabhängige Variable sowie die Kontrollvariablen in das Random-Intercept-Modell auf (Tab. 2, Model A). Zur Prüfung der These, dass die nationalen Bildungsausgaben einen Einfluss auf die Weiterbildungsbeteiligung haben $(\mathrm{H} 2)$, wird das unter A spezifizierte Modell um die Makrovariable der nationalen Bildungsausgaben pro Einwohner ergänzt (Tab. 2, Modell B). Auch hier berechnen wir die ICC. Die Veränderung der Varianzanteile

Tab. 2 Modell Spezifikationen

$$
\begin{array}{ll}
\text { 0 } & \log \left(\frac{N F L_{i j}}{1-N F L_{i j}}\right)=\beta_{0}+u_{j}+e_{i j} \\
\text { A } & \log \left(\frac{N F L_{i j}}{1-N F L_{i j}}\right)=\beta_{0}+\left(\sum_{k=1}^{28} \beta_{k} X_{k i j}\right) u_{j}+e_{i j} \\
\text { B } & \log \left(\frac{N F L_{i j}}{1-N F L_{i j}}\right)=\beta_{0}+\left(\sum_{k=1}^{28} \beta_{k} X_{k i j}\right)+\beta_{29} E X P_{j}+u_{j}+e_{i j} \\
\text { C } & \log \left(\frac{N F L_{i j}}{1-N F L_{i j}}\right)=\beta_{0}+\left(\sum_{k=1}^{28} \beta_{k} X_{k i j}\right)+\beta_{29} E X P_{j}+u_{0 j}+u_{1 j} L O W_{i j}+e_{i j} \\
\text { D } & \log \left(\frac{N F L_{i j}}{1-N F L_{i j}}\right)=\beta_{0}+\left(\sum_{k=1}^{28} \beta_{k} X_{k i j}\right)+\beta_{29} E X P_{j}+\beta_{30} L O W_{i j} E X P_{j}+u_{0 j}+ \\
& u_{1 j} L O W_{i j}+e_{i j}
\end{array}
$$

$N F L$ Non-formales Lernen; Xk Unabhängige Variablen auf der Individualebene; EXP Bildungsausgaben; $L O W$ Geringqualifizierte; $i$ Individualebene; $j$ Länderebene; $u$ und $e$ Residuen; $\beta$ Koeffizient 
zum vorhergehenden Modell ist jedoch nicht umstandslos interpretierbar, da auch bei einer Veränderung des Modells durch zusätzliche Variablen die Varianz der dem logistischen Modell zugrunde liegenden latenten (normalverteilten) Variable auf $\pi^{2} / 3$ festgelegt bleibt (vgl. Hox et al. 2010).

Von zentraler Bedeutung ist die Annahme, dass durch staatliche Ausgaben die Ungleichheit bei der Beteiligung Geringqualifizierter reduziert werden kann (H3). Die Überprüfung dieser These setzt jedoch voraus, dass sich der negative Einfluss dieses Merkmals überhaupt zwischen den Ländern unterscheidet. Dies wird in Modell C (Tab. 2) getestet. Hier wird zu dem länderspezifischen (Intercept) und dem individuellen Fehlerterm zusätzlich ein Fehlerterm aufgenommen, an dem die länderspezifischen Unterschiede der Effektstärke (Slope) des Merkmals Geringqualifiziert abgeschätzt wird. Diese länderspezifischen Effektstärken können in einer zweiten, anschließenden Schätzung (Empirical Bayes Prediction) spezifiziert und dargestellt werden. Dabei werden auf der Grundlage der zuvor geschätzten Verteilung in den Daten die bisher nur als Fehlerterme aufgenommenen länderspezifischen Intercepts und Slopes als Koeffizienten spezifiziert. Diese Log-Odds können als länderspezifische Vor- bzw. Nachteile unabhängig von individuellen Merkmalausprägungen interpretiert werden. Empirical Bayes Predictions zeichnen sich zudem gegenüber ML-Schätzungen durch geringere Schätzfehler aus (vgl. Rabe-Hesketh und Skrondal 2012).

Der länderspezifische Unterschied der Effektstärke geringer Qualifikationen kann dann durch einen Interaktionseffekt zwischen der Makrovariable und dem individuellen Merkmal (Cross Level) erklärt und der Einfluss von Bildungsausgaben auf die Weiterbildungsbenachteiligung Geringqualifizierter ermittelt werden. Dazu wird in das Random-Intercept und Random-Slope Modell C ein entsprechender Interaktionseffekt eingefügt (Tab. 2, Modell D).

Durch Likelihood-Ratio-Tests stellen wir fest, ob die einzelnen Erweiterungen der Modelle eine Verbesserung, also eine größere Passung an die empirischen Daten, darstellen.

\subsection{Ergebnisse}

Wir beschreiben zunächst einige deskriptive Statistiken zu den nationalen Partizipationsstrukturen der Weiterbildung. Anschließend stellen wir die Ergebnisse der zuvor beschriebenen Modelle zum Test der im Theorieteil formulierten Hypothesen vor. An dieser Stelle möchten wir darauf hinweisen, dass jegliche Termini, die auf Kausalität hinweisen, nicht als solche zu interpretieren sind. Mit dem hier auf Querschnittsdaten angewendetem Analyseverfahren können wir keine Aussage zur Kausalität treffen, sondern lediglich Zusammenhänge aufweisen. Die ausgewiesenen Odds sind zudem nicht als direkte, sondern immer nur relative Wahrscheinlichkeiten in Bezug auf eine Basiswahrscheinlichkeit zu interpretieren (vgl. Best und Wolf 2010). 
Abb. 1 Länder- und qualifikationsspezifische Weiterbildungsbeteiligung

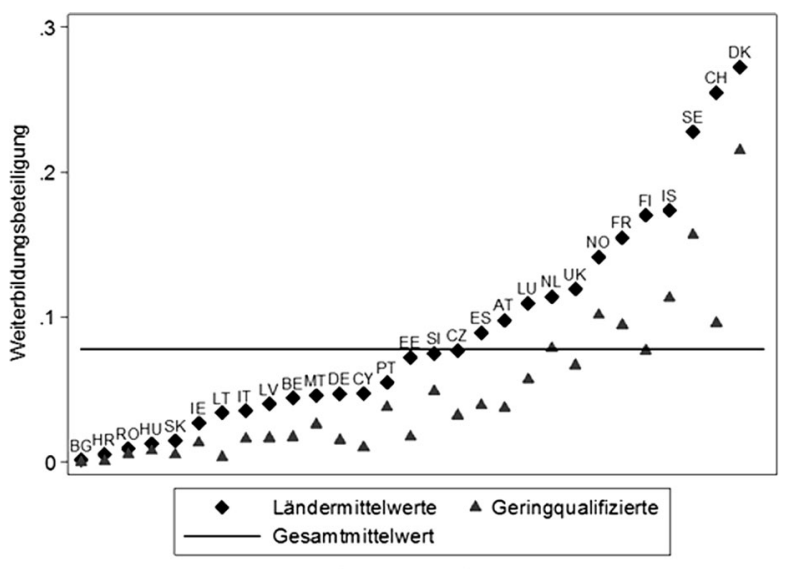

Quelle: EU-LFS 2013

\subsection{Deskriptive Ergebnisse}

Abb. 1 zeigt die Gesamtbeteiligung an Weiterbildung (circa $8 \%$ ) über alle Länder hinweg sowie die Beteiligung innerhalb der betrachteten Länder. Diese sind von links nach rechts geordnet, entsprechend der Höhe der Beteiligung. Das Schlusslicht ist Bulgarien mit einer Beteiligung nahe 0\%. Angeführt wird das Feld von Dänemark mit circa $27 \%$ Beteiligung. Über die Darstellung dieser starken Variation hinaus zeigt die Grafik die Beteiligung Geringqualifizierter, die für alle Länder deutlich unter der Gesamtbeteiligung liegt. Außerdem wird deutlich, dass die Reihenfolge der Länder nach Gesamtbeteiligung nicht übereinstimmt mit der Reihenfolge nach Beteiligung der Geringqualifizierten. Es lässt sich jedoch ein Trend identifizieren, dass in Ländern hoher Beteiligung auch die Beteiligung Geringqualifizierter vergleichsweise hoch ist. Wie weit sie sich von der Gesamtheit abheben und somit, wie stark ihre Benachteiligung ist, unterscheidet sich deutlich über die Länder hinweg.

\subsection{Mehrebenenmodelle}

Tab. 3 beinhaltet die Ergebnisse der Modelle 0, A und B in Form von Log-Odds und deren Standardabweichung. Das Nullmodell (0) dient zur Quantifizierung der Varianzkomponenten von Weiterbildungsbeteiligung, wobei Modell A den Effekt der individuellen Prädiktoren aufzeigt und Modell B durch den Effekt der Makrovariable ergänzt. In logistischen Mehrebenenmodellen ist die Varianz auf IndividualEbene - einer standardisierten logistischen Verteilung folgend - modelltheoretisch festgesetzt auf 3,29 und kann somit nicht über die verschiedenen Modelle hinweg variieren. Die Interpretation von Veränderungen des ICCs, die aus der Aufnahme von Individualmerkmalen resultieren, ist demnach schwierig. Wir werden dennoch dessen klassische Interpretation zur Verfügung stellen und sehen die daraus gewonnen Erkenntnisse als wichtige Richtwerte an. 
Tab. 3 Logistische Mehrebenenregression zur Beteiligung an Weiterbildung (Log-Odds)

\begin{tabular}{|c|c|c|c|c|}
\hline 0 & $\mathrm{~A}$ & & $\mathrm{~B}$ & \\
\hline \multicolumn{5}{|l|}{ Bildung (Referenz: mittel) } \\
\hline Niedrig & $-0,345^{* * *}$ & $(0,01)$ & $-0,345^{* * *}$ & $(0,01)$ \\
\hline Hoch & $0,352^{* * *}$ & $(0,01)$ & $0,352^{* * *}$ & $(0,01)$ \\
\hline Migrant & $-0,222^{* * * *}$ & $(0,01)$ & $-0,222^{* * *}$ & $(0,01)$ \\
\hline \multicolumn{5}{|l|}{ Alter (Referenz: 32 bis 42) } \\
\hline 22 bis 32 & $0,052^{* * *}$ & $(0,01)$ & $0,052^{* * * *}$ & $(0,01)$ \\
\hline 42 bis 52 & $-0,030^{* * * *}$ & $(0,01)$ & $-0,030^{* * *}$ & $(0,01)$ \\
\hline 52 bis 62 & $-0,210^{* * *}$ & $(0,01)$ & $-0,210^{* * *}$ & $(0,01)$ \\
\hline$>62$ & $-0,322^{* * *}$ & $(0,01)$ & $-0,322^{* * *}$ & $(0,01)$ \\
\hline Frau & $0,334^{* * *}$ & $(0,01)$ & $0,334^{* * *}$ & $(0,01)$ \\
\hline Ledig/geschieden/verwitwet & $0,034^{* * *}$ & $(0,01)$ & $0,034^{* * *}$ & $(0,01)$ \\
\hline Kinder im Haushalt & 0,003 & $(0,00)$ & 0,003 & $(0,00)$ \\
\hline $\begin{array}{l}\text { Kinder im Alleinerzie- } \\
\text { her/in-Haushalt }\end{array}$ & $-0,026^{* * *}$ & $(0,00)$ & $-0,026^{* * *}$ & $(0,01)$ \\
\hline Erwerbslos & $0,769^{* * *}$ & $(0,01)$ & $0,769^{* * *}$ & $(0,01)$ \\
\hline Selbstständig & $-0,039^{*}$ & $(0,02)$ & $-0,039^{*}$ & $(0,01)$ \\
\hline Angestellte/r & 0,004 & $(0,02)$ & 0,004 & $(0,02)$ \\
\hline \multicolumn{5}{|c|}{ Anforderungen im Beruf (Referenz: durchschnittlich) } \\
\hline Elementar & $-0,407^{* * *}$ & $(0,02)$ & $-0,407^{* * *}$ & $(0,02)$ \\
\hline Hoch & $0,339^{* * *}$ & $(0,01)$ & $0,339^{* * *}$ & $(0,01)$ \\
\hline Sehr hoch & $0,434^{* * *}$ & $(0,01)$ & $0,434^{* * * *}$ & $(0,01)$ \\
\hline
\end{tabular}

Das Nullmodell (Tab. 3, Modell 0) schätzt die mittlere Weiterbildungsbeteiligung auf circa $5 \%$. Diese Wahrscheinlichkeit $\left(\mathrm{P}_{\mathrm{i}}\right)$ berechnet sich aus der Formel:

$$
P_{i}=\frac{e^{\log \left(\frac{N F L_{i j}}{1-N F L_{i j}}\right)}}{1-e^{\log \left(\frac{N F L_{i j}}{1-N F L_{i j}}\right)}}
$$

Der aus den Varianzkomponenten berechnete ICC weist darauf hin, dass 32,5\% der Gesamtvarianz in der Weiterbildungsbeteiligung auf die nationale Ebene entfallen. In Modell A sinkt dieser auf knapp $31 \%$. Die auf individueller Ebene festgelegte Varianz schließt eine Interpretation der Varianzaufklärung durch individuelle Merkmale über die Ebenen aus.

Modell A zeigt deutlich den erwarteten Zusammenhang zwischen Qualifikation durch Bildung und Weiterbildungsbeteiligung. Unter Konstanthalten aller Kontrollvariablen sind die Log-Odds an Weiterbildung teilzunehmen für Individuen mit niedriger Bildung signifikant negativ, während diese für höher Gebildete signifikant positiv sind. Den Referenzpunkt bilden Individuen mit mittlerer Bildung. Die Odds ( $\left.\mathrm{e}^{\log -\text { odds }}\right)$, an Weiterbildung teilzunehmen, sind für Geringqualifizierte $29,14 \%$ geringer als für Individuen mit mittlerer Bildung. Die Log-Odds der Kontrollvariablen bestätigen bestehende Erkenntnisse. 
Tab. 3 Logistische Mehrebenenregression zur Beteiligung an Weiterbildung (Log-Odds) (Fortsetzung)

\begin{tabular}{|c|c|c|c|c|c|c|}
\hline & 0 & & $\mathrm{~A}$ & & $\mathrm{~B}$ & \\
\hline \multicolumn{7}{|c|}{ Berufsklassifikation (Referenz: (2)) } \\
\hline (1) & & & $-0,072^{* * *}$ & $(0,02)$ & $-0,072^{* * *}$ & $(0,02)$ \\
\hline (3) & & & $0,303^{* * *}$ & $(0,02)$ & $0,303^{* * *}$ & $(0,02)$ \\
\hline (4) & & & $-0,163^{* * *}$ & $(0,02)$ & $-0,163^{* * *}$ & $(0,02)$ \\
\hline (5) & & & $-0,003$ & $(0,01)$ & $-0,003$ & $(0,01)$ \\
\hline (6) & & & $-0,374^{* * *}$ & $(0,02)$ & $-0,374^{* * *}$ & $(0,02)$ \\
\hline (7) & & & $0,131^{* * *}$ & $(0,02)$ & $0,131^{* * *}$ & $(0,02)$ \\
\hline (8) & & & $0,058^{* * *}$ & $(0,02)$ & $0,058^{* * * *}$ & $(0,02)$ \\
\hline (9) & & & $0,325^{* * *}$ & $(0,01)$ & $0,325^{* * *}$ & $(0,01)$ \\
\hline (10) & & & $-0,090^{*}$ & $(0,04)$ & $-0,091^{*}$ & $(0,04)$ \\
\hline $\begin{array}{l}\text { Betriebsgröße }>10 \text { Mitarbei- } \\
\text { ter }\end{array}$ & & & $0,170^{* * * *}$ & $(0,01)$ & $0,170^{* * * *}$ & $(0,01)$ \\
\hline Arbeitszeit & & & $0,007^{* * *}$ & $(0,01)$ & $0,007^{* * * *}$ & $(0,00)$ \\
\hline Bildungsausgaben & & & & & $0,835^{* * *}$ & $(0,17)$ \\
\hline Konstante & $-2,8571^{* * *}$ & $(0,23)$ & $-3,557^{* * * *}$ & $(0,22)$ & $-3,556^{* * *}$ & $(0,16)$ \\
\hline \multicolumn{7}{|l|}{ Varianz-Komponenten } \\
\hline Individual-Ebene & 3,29 & & 3,29 & & 3,29 & \\
\hline Länder-Ebene & 1,5855 & & 1,4646 & & 0,7829 & \\
\hline ICC & 0,3252 & & 0,3080 & & 0,1922 & \\
\hline AIC & 1.219 .191 & & 1.139 .527 & & 1.139 .511 & \\
\hline BIC & 1.219 .217 & & 1.139 .910 & & 1.139 .906 & \\
\hline LRT $\left(\operatorname{chi}^{2}(\Delta\right.$ Freiheitsgrade $\left.)\right)$ & & & $79.720(28)^{* * * *}$ & & $19(1)^{* * *}$ & \\
\hline Beobachtungen & 2.550 .763 & & 2.550 .763 & & 2.550 .763 & \\
\hline Länder & 30 & & 30 & & 30 & \\
\hline
\end{tabular}

Quelle: EU-LFS 2013

Standardabweichung in Klammern; ${ }^{*} p<0,05,{ }^{* *} p<0,01,{ }^{* * *} p<0,001$

Modell B erweitert Modell A um die unabhängige Makrovariable der öffentlichen Bildungsausgaben. Diese sind um den Mittelwert standardisiert. Der direkte Effekt auf die Weiterbildungsbeteiligung ist positiv und signifikant. Dessen Stärke ist beachtlich. Mit den Log-Odds von circa 0,8 ist der Zusammenhang zwischen einer Erhöhung der Bildungsausgaben um eine Standardabweichung und der Beteiligung an Weiterbildung stärker als jeder andere gemessene Zusammenhang auf der Individualebene. Die Erhöhung der Bildungsausgaben um eine Standardabweichung führt zu einem Anstieg der Odds zur Teilnahme um $130 \%$, womit sich die Odds mehr als verdoppeln. Die ICC sinkt auf 19\%. Somit kann ein Drittel der Varianz in der Weiterbildungsbeteiligung zwischen den untersuchten Ländern durch die Höhe der Bildungsausgaben erklärt werden.

In Tab. 4 werden die Ergebnisse der Modelle $\mathrm{C}$ und D vorgestellt, ebenfalls in Form von Log-Odds und deren Standardabweichung. Die Modelle unterscheiden sich von den bisherigen durch eine weitere Varianzkomponente. Sie erlauben eine Variation des Effektes niedriger Bildung innerhalb der Länder. Diese Variation ist signifikant. $\mathrm{Zu}$ interpretieren ist sie in der Form, dass wir in $95 \%$ der Länder im europäischen Raum (von denen die von uns analysierten 30 Länder eine Stichpro- 


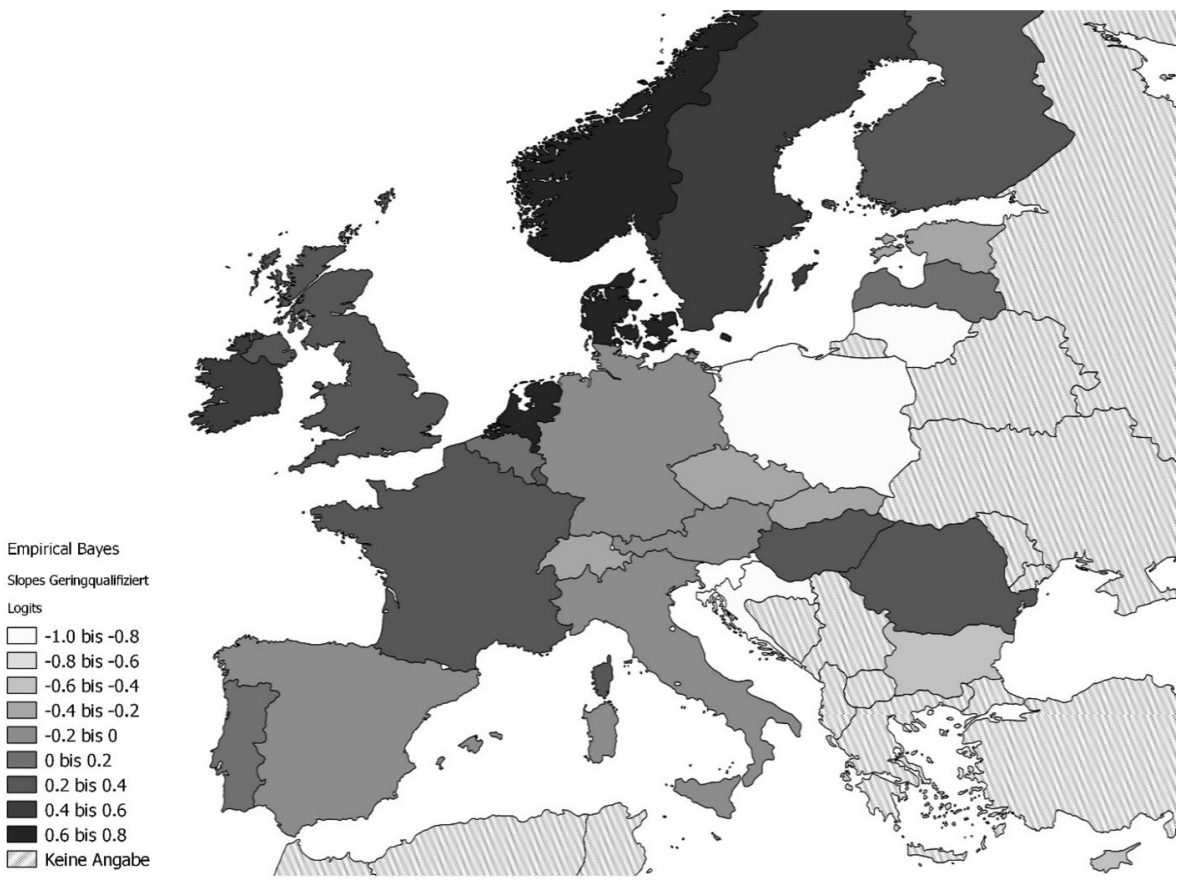

Abb. 2 Empirical Bayes: länderspezifische Slopes der Weiterbildungsbeteiligung Geringqualifizierter (Log-Odds)

Tab. 4 Logistische Mehrebenenregression zur Beteiligung an Weiterbildung (Log-Odds)

\begin{tabular}{lllll}
\hline & $\mathrm{C}$ & & $\mathrm{D}$ & \\
\hline Bildung (Referenz: mittel) & & & & \\
Niedrig & $-0,596^{* * *}$ & $(0,08)$ & $-0,599^{* * *}$ & $(0,07)$ \\
Hoch & $0,379^{* * *}$ & $(0,01)$ & $0,379^{* * * *}$ & $(0,01)$ \\
Migrant & $-0,218^{* * *}$ & $(0,01)$ & $-0,218^{* * *}$ & $(0,01)$ \\
Alter (Referenz: 32 bis 42) & & & \\
22 bis 32 & $0,054^{* * *}$ & $(0,01)$ & $0,054^{* * *}$ & $(0,01)$ \\
42 bis 52 & $-0,037^{* * *}$ & $(0,01)$ & $-0,037^{* * * *}$ & $(0,01)$ \\
52 bis 62 & $-0,221^{* * *}$ & $(0,01)$ & $-0,221^{* * *}$ & $(0,01)$ \\
62 & $-0,330^{* * *}$ & $(0,01)$ & $-0,330^{* * *}$ & $(0,01)$ \\
Frau & $0,335^{* * *}$ & $(0,01)$ & $0,335^{* * * *}$ & $(0,01)$ \\
Ledig/geschieden/verwitwet & $0,027^{* * *}$ & $(0,00)$ & $0,027^{* * * *}$ & $(0,01)$ \\
Kinder im Haushalt & 0,001 & $(0,00)$ & 0,001 & $(0,00)$ \\
Kinder im Alleinerzieher/in-Haushalt & $-0,023^{* * *}$ & $(0,00)$ & $-0,023^{* * *}$ & $(0,00)$ \\
Erwerbslos & $0,762^{* * *}$ & $(0,01)$ & $0,762^{* * *}$ & $(0,01)$ \\
Selbstständig & $-0,071^{* * *}$ & $(0,02)$ & $-0,071^{* * *}$ & $(0,02)$ \\
Angestellte/r & $-0,021$ & $(0,02)$ & $-0,021$ & $(0,02)$ \\
Anforderungen im Beruf (Referenz: durchschnittlich) & & & \\
Elementar & $-0,376^{* * *}$ & $(0,02)$ & $-0,376^{* * *}$ & $(0,02)$ \\
Hoch & $0,336^{* * *}$ & $(0,01)$ & $0,336^{* * * *}$ & $(0,01)$ \\
Sehr hoch & $0,448^{* * *}$ & $(0,01)$ & $0,448^{* * * *}$ & $(0,01)$ \\
\hline
\end{tabular}


Tab. 4 Logistische Mehrebenenregression zur Beteiligung an Weiterbildung (Log-Odds) (Fortsetzung)

\begin{tabular}{|c|c|c|c|c|}
\hline & $\mathrm{C}$ & & $\mathrm{D}$ & \\
\hline \multicolumn{5}{|l|}{ Berufsklassifikation (Referenz: (2)) } \\
\hline (1) & $-0,041$ & $(0,02)$ & $-0,040$ & $(0,02)$ \\
\hline (3) & $0,298^{* * *}$ & $(0,02)$ & $0,298^{* * *}$ & $(0,02)$ \\
\hline (4) & $-0,167^{* * *}$ & $(0,02)$ & $-0,167^{* * *}$ & $(0,02)$ \\
\hline (5) & $-0,005$ & $(0,01)$ & $-0,005$ & $(0,01)$ \\
\hline (6) & $-0,356^{* * *}$ & $(0,02)$ & $-0,356^{* * *}$ & $(0,02)$ \\
\hline (7) & $0,126^{* * *}$ & $(0,02)$ & $0,126^{* * *}$ & $(0,02)$ \\
\hline (8) & $0,061^{* * *}$ & $(0,02)$ & $0,061^{* * *}$ & $(0,02)$ \\
\hline (9) & $0,320^{* * *}$ & $(0,01)$ & $0,320^{* * *}$ & $(0,01)$ \\
\hline$(10)$ & $-0,067$ & $(0,04)$ & $-0,067$ & $(0,04)$ \\
\hline Betriebsgröße >10 Mitarbeiter & $0,171^{* * *}$ & $(0,01)$ & $0,171^{* * *}$ & $(0,01)$ \\
\hline Arbeitszeit & $0,007^{* * *}$ & $(0,00)$ & $0,007^{* * *}$ & $(0,00)$ \\
\hline Bildungsausgaben & $0,774^{* * *}$ & $(0,16)$ & $0,772^{* * *}$ & $(0,16)$ \\
\hline Niedrige Bildung*Bildungsausgaben & & & $0,277^{* * *}$ & $(0,07)$ \\
\hline Konstante & $-3,518^{* * *}$ & $(0,16)$ & $-3,517^{* * *}$ & $(0,16)$ \\
\hline \multicolumn{5}{|l|}{ Varianz-Komponenten } \\
\hline Individual-Ebene & 3,29 & & 3,29 & \\
\hline Länder-Ebene & 0,7700 & & 0,7696 & \\
\hline Bildung: niedrig & 0,2148 & & 0,1379 & \\
\hline ICC & 0,1897 & & 0,1896 & \\
\hline AIC & 1.136 .660 & & 1.136 .649 & \\
\hline $\mathrm{BIC}$ & 1.137 .068 & & 1.137 .070 & \\
\hline LRT $\left(\operatorname{chi}^{2}(\Delta\right.$ Freiheitsgrade $\left.)\right)$ & $2853(1)^{* * *}$ & & $12(1)^{* * *}$ & \\
\hline Beobachtungen & 2.550 .763 & & 2.550 .763 & \\
\hline Länder & 30 & & 30 & \\
\hline
\end{tabular}

Quelle: EU-LFS 2013

Standardabweichung in Klammern; ${ }^{*} p<0,05,{ }^{* *} p<0,01,{ }^{* * *} p<0,001$

be sind) Log-Odds für niedrige Bildung zwischen -1,5045 und 0,3120 erwarten $\left(\beta_{\mathrm{LOW}} \pm 1,96 * \operatorname{sd}(\mathrm{LOW})\right)$. Diese Effektspanne verdeutlicht, dass somit Geringqualifizierte nicht in allen Ländern benachteiligt sind in Bezug auf die Weiterbildungsbeteiligung, sondern gleichberechtigt oder sogar bevorzugt sind. Der negative Bereich ist jedoch deutlich größer als der positive und somit ist die Benachteiligung wahrscheinlicher als die anderen beiden Fälle. Abb. 2 verdeutlicht die Verteilung der Effekte geringer Qualifikationen im europäischen Raum anhand der durch Empirical Bayes geschätzten Länder-Koeffizienten.

Zur Erklärung dieses spezifischen Varianzanteils wird in Modell D ein Interaktionsterm zwischen niedriger Bildung und den Bildungsausgaben hinzugefügt. Dieser ist signifikant positiv. Das bedeutet, dass der negative Effekt niedriger Bildung auf die Weiterbildungsbeteiligung schwächer wird bei höheren Bildungsausgaben. Genauer führt eine Erhöhung der Bildungsausgaben um eine Standardabweichung zu einer Verringerung der Log-Odds für niedrig Qualifizierte auf $-0,599+0,277=$ $-0,322$. Betrachtet man den Unterschied zwischen den Varianztermen niedriger Bildung von Modell C und D, lässt sich eine starke Reduktion beobachten. Bildungsausgaben erklären 36\% dieser Varianz. Da sich die Modelle nur hinsichtlich einer 
Makrovariablen unterscheiden, interpretieren wir dies als einen deutlichen Effekt. Wie bereits erwähnt ist die Veränderung der ICC jedoch nicht klar als Quantität aufgeklärter Varianz interpretierbar.

Um sicherzustellen, dass durch die Modellerweiterungen eine höhere Passung an die empirischen Daten gegeben wird, führen wir Likelihood-Ratio-Tests durch, die auf Basis der Chi-Square-Statistik die Modelle untereinander vergleichen. Die Testergebnisse sind in den Spalten der jeweiligen Modellerweiterung (Tab. 3 und 4) aufgeführt.

\section{Zusammenfassung und Diskussion}

Ausgangspunkt dieses Artikels ist die Forschungsfrage, welchen Einfluss nationale Kontexte und Regulative auf die Weiterbildungsbeteiligung tatsächlich haben und inwiefern es auf dieser Handlungsebene gelingt, die Partizipation von Bevölkerungsgruppen, deren Beteiligungschancen durch typische Barrieren eingeschränkt sind, zu erhöhen und so soziale Selektivität im Bildungsverhalten abzubauen. Anhand von handlungs- und regimetheoretischen Überlegungen formulierten wir drei Hypothesen, die den Zusammenhang zwischen niedriger individueller Bildung, nationalen Bildungsausgaben und individueller Weiterbildungsbeteiligung spezifizierten. Diese testeten wir mit verschiedenen logistischen Mehrebenenmodellen, angewendet auf Daten des EU-LFS 2013.

Die Ergebnisse machen deutlich, dass es auf der Ebene der Nationalstaaten erhebliche Steuerungspotenziale gibt. Mit einer ICC von über 0,3 ist die Bedeutung nationalstaatlicher Gebietskörperschaften erheblich größer, als dies bisher für regionale und kommunale Räume nachgewiesen werden konnte. Die Bedeutung wird noch verstärkt, wenn man berücksichtigt, dass hier nur europäische Länder untersucht wurden, von denen 28 Länder Mitglieder der Europäischen Union sind. Es ist seit Langem ein erklärtes Ziel europäischer Politik, die Unterschiede zwischen den Ländern zu harmonisieren. Die Schätzung der Relevanz nationalstaatlicher Gebietskörperschaften ist also eher konservativ.

Ein Drittel der Varianz am Weiterbildungsverhalten auf Länderebene lässt sich durch die nationalen Bildungsausgaben erklären. Auch wenn dieser Varianzanteil schwer zu interpretieren ist, wird Hypothese 2 bestätigt. Neben weiteren möglichen kulturellen, sozialen und wirtschaftlichen Einflüssen ist auch die nationale Bildungspolitik ein wichtiger Faktor. Sie ist in der Lage, das Weiterbildungsverhalten direkt oder indirekt durch finanzielles Engagement zu steuern.

Diese Handlungsfähigkeit wird umso deutlicher, wenn der Einfluss bildungspolitischer Interventionen auf das Weiterbildungsverhalten von typisch benachteiligten Personen untersucht wird. Wie zu erwarten (H1), weisen Geringqualifizierte auch im internationalen Rahmen und unter Kontrolle zentraler sozioökonomischer und demografischer Merkmale eine geringere Teilnahmewahrscheinlichkeit im Vergleich zur Wohnbevölkerung mit mittlerer Bildung auf. Zentral ist jedoch, dass dieser Effekt in den europäischen Ländern signifikant unterschiedlich ausgeprägt ist. Dies bedeutet, dass der Nachteil einer Person mit geringer Qualifikation zur Partizipation an Weiterbildung davon abhängt, in welchem Land diese Person lebt. Anhand des Em- 
pirical Bayes zeigt sich, dass diese Nachteile in skandinavischen Ländern auch unter Kontrolle sozioökonomischer und demografischer Merkmale auf der Individualebene am geringsten sind. Nicht ganz erwartungswidrig und in Übereinstimmung mit Annahmen der Wohlfahrtsregimetheorie ist die Ungleichheit der Bildungschancen in diesen eher sozialdemokratisch geprägten Ländern am geringsten ausgeprägt.

Aus dieser theoretischen Überlegung ergab sich auch die Annahme, dass der Einfluss nationaler Kontexte und Regulative auf die Benachteiligung von Geringqualifizierten durch öffentliche Bildungsausgaben erklärt werden kann. Dies basiert auf der Prämisse, dass de-kommodifizierende Aktivitäten des Staates der Kumulation von Ungleichheiten in marktvermittelten Zusammenhängen entgegenwirken. Je höher also die öffentlichen Bildungsausgaben, umso geringer sollten die Nachteile von Geringqualifizierten in Bezug auf die Weiterbildungsbeteiligung sein (H3). Auch diese Annahme wurde bestätigt. Öffentliche Bildungsausgaben können demnach tatsächlich der marktvermittelten Kumulation von Bildungschancen entgegenwirken. Der Staat ist also auch in diesem sehr spezifischen Bereich ein handlungsfähiger Akteur.

Zusammenfassend erweist sich die nationalstaatliche Handlungsebene als ein relevanter Kontext des Weiterbildungsverhaltens und setzt sich darin von regionalen und kommunalen Gebietskörperschaften ab. Zudem ist der Staat auf dieser Ebene in der Lage, sowohl die Effektivität (in Form von Beteiligung) als auch die Chancengleichheit dieses Bildungsbereichs zu beeinflussen.

Diese Resultate konterkarieren jedoch neuere Steuerungskonzepte im Zusammenhang mit der Educational Governance, in denen Dezentralisierung und die Bedeutung regionaler und kommunaler Koordinationsrahmen sowie die Ausrichtung von Steuerungsaktivitäten an den Outcomes betont werden. Stattdessen hat sich in unserer Untersuchung der "traditionelle“ Handlungsrahmen der Nationalstaaten als relevant und die denkbar einfachste Form der Inputsteuerung (Finanzierung) als effektiv erwiesen.

Da unsere Studie jedoch über korrelative Zusammenhänge hinaus wenig Aussagekraft besitzt, empfehlen wir zukünftiger Forschung die längsschnittliche Betrachtung dieser Zusammenhänge. Die Erhebung entsprechender Daten sollte im Fokus der Wissenschaft liegen. Die Operationalisierung der Weiterbildungsbeteiligung ist im EU-LFS zudem eher undifferenziert und lässt keine systematischen Aussagen zu den einzelnen Segmenten der Weiterbildung zu. Spezifischer sollte auch die kontextuelle ökonomische Situation der Individuen in Folgestudien mit einbezogen werden. Auch die Untersuchung möglicher Kovarianz zwischen Intercept und Slope kann zu weiteren wichtigen Erkenntnissen führen. Letztlich bietet diese Studie große Gelegenheit zur Replikation für andere benachteiligte Gruppen wie Migranten oder Ältere.

Open Access Dieser Artikel wird unter der Creative Commons Namensnennung 4.0 International Lizenz (http://creativecommons.org/licenses/by/4.0/deed.de) veröffentlicht, welche die Nutzung, Vervielfältigung, Bearbeitung, Verbreitung und Wiedergabe in jeglichem Medium und Format erlaubt, sofern Sie den/die ursprünglichen Autor(en) und die Quelle ordnungsgemäß nennen, einen Link zur Creative Commons Lizenz beifügen und angeben, ob Änderungen vorgenommen wurden. 


\section{Literatur}

Bassanini, A., Booth, A. L., Brunello, G., De Brunello Paola, M., \& Leuven, E. (2005). Workplace training in Europe. IZA Discussion Papers, 1640.

Behringer, F., \& Schönfeld, G. (2014). C Schwerpunktthema: Lernen Erwachsener in Deutschland im europäischen Vergleich. In Bundesinstitut für Berufsbildung (BIBB) (Hrsg.), Datenreport zum Berufsbildungsbericht 2014. Informationen und Analysen zur Entwicklung der beruflichen Bildung (S. 381-413). Bielefeld: W. Bertelsmann.

Best, H., \& Wolf, C. (2010). Logistische Regression. In C. Wolf \& H. Best (Hrsg.), Handbuch der sozialwissenschaftlichen Datenanalyse (S. 827-854). Wiesbaden: VS Verlag für Sozialwissenschaften.

Bilger, F., \& Kuper, H. (2013). Trendvergleich: Teilnahme und Aktivitäten. In F. Bilger, D. Gnahs, J. Hartmann, \& H. Kuper (Hrsg.), Weiterbildungsverhalten in Deutschland. Resultate des Adult Education Survey 2012 (S. 95-103). Bielefeld: W. Bertelsmann.

BMBF - Bundesministerium für Bildung und Forschung (2008). Berufsbildungsbericht 2008. Bielefeld: W. Bertelsmann.

Boeren, E., \& Holford, J. (2016). Vocationalism varies (a lot) A 12-country multivariate analysis of participation in formal adult learning. Adult Education Quarterly. doi:10.1177/0741713615624207.

Boeren, E., Holford, J., Nicaise, I., \& Baert, H. (2012). Why do adults learn? Developing a motivational typology across twelve European countries. Globalisation, Societies and Education, 10, 247-269.

Bourdieu, P. (1990). The logic of practice. Stanford: Stanford University Press.

Castles, F. G., \& Mitchell, D. (1993). Worlds of welfare and families of nations. In F. G. Castles (Hrsg.), Families of nations: patterns of public policy in western democracies (S. 93-128). Aldershot: Dartmouth.

Castles, F. G., \& Obinger, H. (2008). Worlds, families, regimes: country clusters in European and OECD area public policy. West European Politics, 31(1/2), 321-344.

Cross, K.P. (1981). Adults as learners. Increasing participation and facilitating learning. San Francisco: Jossey-Bass.

Dämmrich, J., Vono, D., \& Reichart, E. (2014). Participation in adult learning in Europe: the impact of country-level and individual characteristics. In H.-P. Blossfeld, E. Kilpi-Jakonen, D. Vono de Vilhena \& S. Buchholz (Hrsg.), Adult learning in modern societies: an international comparison from a lifecourse perspective (S. 29-55). Cheltenham: Edward Elgar.

Desjardins, R., \& Rubenson, K. (2013). Participation patterns in adult education: the role of institutions and public policy frameworks in resolving coordination problems. European Journal of Education, $48(2), 262-280$.

Dieckhoff, M., Junglbut, J.-M., \& O’Connell, P. J. (2007). Job-related training in Europe: Do institutions matter? In D. Gallie (Hrsg.), Employment regimes and the quality of work (S. 77-104). Oxford: Oxford University Press.

Ebbinghaus, B. (2012). Comparing welfare state regimes: Are typologies an ideal or realistic strategy? European Social Policy Analysis Network, ESPAnet Conference, Edinburgh, 6-8 September. Vol. 120.

Ericson, T. (2005). Personnel training: a theoretical and empirical review. Working paper series from IFAU - Institute for Evaluation of Labour Market and Education Policy. http://www.ifau.se/upload/ pdf/se/2005/wp05-01.pdf. Zugegriffen: 26. April 2016.

Esping-Andersen, G. (1990). Three worlds of welfare capitalism. Princeton: Princeton University Press.

Esping-Andersen, G. (1999). Social foundations of postindustrial economies. Oxford: Oxford University Press.

Europäische Union (2011). Entschließung des Rates über eine erneuerte europäische Agenda für die Erwachsenenbildung. http://eurlex.europa.eu/LexUriServ/LexUriServ.do?uri=OJ:C:2011:372:0001: 0006:de:PDF. Zugegriffen: 21. April 2016.

Eurostat (2015). User guide. EU labour force survey database. http://ec.europa.eu/eurostat/documents/ 1978984/6037342/EULFS-Database-UserGuide.pdf. Zugegriffen: 28. Mai 2016

Eurostat (2016). Education finance statistics. Table 1: Main indicators for public expenditure on education (excluding early childhood educational development). http://ec.europa.eu/eurostat/ statisticsexplained/index.php/File:Main_indicators_for_public_expenditure_on_education_ \%28excluding_early_childhood_educational_development\%29,_2012_ET15.png. Zugegriffen: 28. Mai 2016.

Goldstein, H., Browne, W., \& Rasbash, J. (2002). Partitioning variation in multilevel models. Understanding Statistics, 1(4), 223-231. 
Hall, P. A., \& Soskice, D. (2001). Varieties of capitalism: the institutional foundations of comparative advantage. Oxford: Oxford University Press.

Hox, J. J., Moerbeek, M., \& van de Schoot, R. (2010). Multilevel analysis: techniques and applications. New York: Routledge.

Kaufmann, K., Reichart, E., \& Schömann, K. (2014). Der Beitrag von Wohlfahrtsstaatsregimen und Varianten kapitalistischer Wirtschaftssysteme zur Erklärung von Weiterbildungsteilnahmestrukturen bei Ländervergleichen. REPORT, 37(2), 39-54.

Kulke, E. (2013). Wirtschaftsgeografie. Paderborn: Schöningh UTB.

Kuper, H., Unger, K., \& Hartmann, J. (2013). Multivariate Analysen zur Weiterbildungsbeteiligung. In F. Bilger, D. Gnahs, J. Hartmann \& H. Kuper (Hrsg.), Weiterbildungsverhalten in Deutschland. Resultate des Adult Education Survey 2012 (S. 95-103). Bielefeld: W. Bertelsmann.

Langer, W. (2009). Mehrebenenanalyse. Wiesbaden: VS.

Markowitsch, J., Käpplinger, B., \& Hefler, G. (2013). Firm-provided training in europe and the limits of national skills strategies. European Journal of Education, 48(2), 281-291.

Martin, A., \& Schömann, K. (2015). Zur Relevanz regionaler und kommunaler Akteurskonstellationen für das Weiterbildungsverhalten. In M. Schemmann (Hrsg.), Internationales Jahrbuch der Erwachsenenbildung (Bd. 38, S. 153-176). Köln: Böhlau.

Martin, A., Schömann, K., \& Schrader, J. (2016). Der Einfluss der kommunalen Steuerung auf Weiterbildungsteilnehmende - Ein Mehrebenen Modell mit Daten des Mikrozensus in Deutschland. Zeitschrift für Erziehungswissenschaft, (1), 55-82.

Martin, A., Schömann, K., Schrader, J., \& Kuper, H. (Hrsg.). (2015). Deutscher Weiterbildungsatlas. Bielefeld: W. Bertelsmann.

Peters, M. A., Besley, Z., \& Araya, D. (2014). Introduction: the new development paradigm: education, knowledge economy, and digital futures. In M. A. Peters, Z. Besley \& D. Araya (Hrsg.), The new development paradigm. Education, knowledge economy, and digital futures. New York: Peter Lang.

Rabe-Hesketh, S., \& Skrondal, A. (2012). Categorical responses counts and survival (3. Aufl.). Multilevel and longitudinal modeling using stata, Bd. 2. College Station: Stata Press.

Rammstedt, B. (2013). Grundlegende Kompetenzen Erwachsener im internationalen Vergleich: Ergebnisse von PIAAC 2012. Münster: Waxmann.

Reutter, G. (2011). Berufliche Weiterbildung für „Geringqualifizierte“: (Fehl-)Investition in Humankapital mit unzureichenden Renditeerwartungen? In G. Niedermair (Hrsg.), Aktuelle Trends in der beruflichen Aus- und Weiterbildung: Impulse, Perspektiven und Reflexionen (S. 245-258). Linz: Trauner.

Roosmaa, E.L., \& Saar, E. (2012). Participation in non-formal learning in EU-15 and EU-8 countries: demand and supply side factors. International Journal of Lifelong Education, 31(4), 477-501.

Rubenson, K., \& Desjardins, R. (2009). The impact of welfare state regimes on barriers to participation in adult education a bounded agency model. Adult education quarterly, 59(3), 187-207.

Schiener, J., Wolter, F., \& Rudolphi, U. (2013). Weiterbildung im betrieblichen Kontext. In R. Becker \& A. Schulze (Hrsg.), Bildungskontexte. Strukturelle Voraussetzungen und Ursachen ungleicher Bildungschancen (S. 555-594). Wiesbaden: Springer VS.

Schrader, J. (2008). Steuerung im Mehrebenensystem der Weiterbildung - ein Rahmenmodell. In J. Schrader \& S. Hartz (Hrsg.), Steuerung und Organisation in der Weiterbildung (S. 31-64). Bad Heilbrunn: Klinkhardt.

Schrader, J. (2011). Struktur und Wandel der Weiterbildung. Bielefeld: W. Bertelsmann.

Siebert, H. (2006). Lernmotivation und Bildungsbeteiligung. Bielefeld: W. Bertelsmann.

Solga, H. (2005). Ohne Abschluss in die Bildungsgesellschaft. Die Erwerbschancen gering qualifizierter Personen aus soziologischer und ökonomischer Perspektive. Opladen: Budrich.

Wolbers, M. H. J. (2005). Initial and Further Education: Substitutes or Complements? Differences in Continuing Education and Training over the Life-Course of European Workers. International Review of Education, 51(5/6), 459-478. 